\title{
An Environmental Model of Self-Compatibility Transitions in the Solanaceae Plant Family
}

\author{
Paul Calcraft ${ }^{1}$, Phil Husbands ${ }^{1}$, and Andrew Philippides ${ }^{1}$ \\ ${ }^{1}$ Centre for Computational Neuroscience and Robotics, Department of Informatics, University of Sussex \\ P.Calcraft@sussex.ac.uk
}

\begin{abstract}
Higher level selection processes such as species selection are not generally predicted to overpower individual selection on character traits. Goldberg et al. provide a model derived from collected life history data and argue that species selection is maintaining self-incompatibility in the Solanaceae plant family. This model applies only on the level of the species, not representing the underlying interactions between individuals and the environment. We propose a new model with environmental variation at the individual level that may explain the maintenance and frequency of loss of this character trait. We use individual based modelling techniques to explore our hypothesis, and compare it with that originally proposed. The results show alternative values required for the mutation rate to produce the species level transition frequency under the opposing models, given certain assumptions. Future work is suggested to refine the parameter relationships, test for robustness, and determine if individual models of higher complexity will exhibit similar outcomes.
\end{abstract}

\section{Introduction}

Evolutionary questions that address multiple levels of the biological hierarchy offer a particular challenge to researchers. There is lack of consensus among biologists about the level(s) at which Darwinian natural selection should be considered to act (Okasha, 2006). This debate about the levels of selection has a complex history, marked by the group selection controversy (Wilson, 1983; Okasha, 2001), and theories of multi-level selection (Damuth and Heisler, 1988) and inclusive fitness (Hamilton, 1964; Queller, 1992).

Empirical data concerning the life history of individuals and species is in many cases insufficient to answer biological questions conclusively (Johnson and Omland, 2004; Turchin, 2013). Mathematical models have been used extensively, but they cannot capture the complexity of interactions in all cases. Individual based modelling techniques can be used to approach problems of this nature, using computer simulations of interacting systems at multiple levels. These models can expose outcomes of theoretical positions that may not be readily apparent (Di Paolo et al., 2000). Their flexibility and speed additionally allow systematic exploration of parameter spaces, testing the robustness and plau- sibility of proposed ideas. Their potential for incorporating environmental interaction can be key in exposing the workings of natural systems (Brooks, 1991).

In this paper we model the individual interactions within the species that underlie the model of Goldberg et al. (2010). We consider individual level selection, that is, natural selection competing between individuals within a given species, to try to expose the lower level dynamics that are giving rise to the outcomes seen at the level of species. We compare two alternative formulations with different profiles of environmental interaction; the first assuming that, as proposed by Goldberg et al., species selection is acting in direct opposition to the lower level individual selection; the second introducing environmental variation. Individual level selection processes are generally considered more powerful than their higher level counterparts such as species selection (Lewontin, 1970). Species selection is therefore rarely cited as able to maintain a trait that is disadvantageous to the individual, but this has been suggested in the case of selfincompatibility in the Solanaceae (nightshade) plant family (Goldberg et al., 2010).

In the next section we discuss the evolutionary background of self-incompatibility in the context of Goldberg et al.'s work. We then introduce the theoretical concepts behind the competing evolutionary incentives. Following this, we lay out the mathematical model of fitness that will form the basis of our individual model. With the mathematical framework in place, we describe the details of the computer simulation and the relevant parameters. Finally, we go on to discuss the results.

\section{Self-Incompatibility and the Goldberg et al. Model}

Self-incompatibility (SI) in plants is a mechanism to prevent self-fertilisation and encourage outcrossing - reproduction with those genetically dissimilar; this increases the genetic diversity of offspring (Barrett, 1988). The alternative, however - self-compatibility (SC) - can be immediately evolutionarily advantageous to individuals in SI populations, as self-fertilising (or selfing) allows plants to pass on their 
genes with higher probability, and selfing plants need not rely on inbound pollen when it is scarce (Lloyd, 1992; Igic et al., 2008).

Goldberg et al. reconstructed a tree of life for the nightshade family, and found a maximum likelihood model that captures the evolution of the species in the family and their relationship with self-incompatibility and self-compatibility. The model shows that SI species have an average rate of transition to SC of 0.555 per lineage per million years, yet a proportion of SI species continues to survive over evolutionary time. This appears to be because SI species have a higher net rate of growth than SC species. This difference (a species-level advantage) is greater than the rate of transition, allowing a proportion of species to be maintained as SI ongoing, despite regular transition to SC.

In claiming that species selection is maintaining selfincompatibility, Goldberg et al. argue that individual evolutionary incentive for $\mathrm{SC}$ is constantly present, but the rate of arrival (and spreading) of the SC mutation in each SI population is sufficiently low to keep the transition rate averaging at this 0.555 per million years figure. This rate is low enough to allow the difference in net species growth (diversification) to be the more significant evolutionary force.

We propose a model where a background factor, a rate of occurrence of temporary environmental disruption, is the cause for a given species transition to SC. Under this model, individual selection does not constantly favour selfing, but is rather selectively neutral, or marginally favouring outcrossing. When the environmental disruption occurs, the relative fitness of selfers increases temporarily, and there is opportunity for an SC mutant to arrive and spread in the population. As long as this model can be shown to achieve the same average transition rate for reasonable sets of parameters, it may offer an alternative explanation for the maintenance of outcrossing that does not require species selection to overpower individual selection unaided.

It is important to note that while transitions from SI to SC are regular and frequent, transitions back to SI are negligibly rare (Igic et al., 2008). This may be due to the complexity of the SI system; it requires genetic coordination at many loci, so there are many points of failure (Franklin-Tong, 2008). There may also be a self-perpetuating dynamic to selfer evolution, as under certain conditions, an increase in the proportion of selfers also increases selfer fitness, making evolution back to SI once a species has been fully invaded by SC particularly improbable.

\section{Individual Selection Models of Selfing versus Outcrossing}

A strong individual incentive for selfing is believed to be its transmission advantage, termed automatic selection (Fisher, 1941). Selfers have a 3:2 advantage of gene transmission, as their seeds on average contribute two gametes to the next generation to the outcrosser's one (while both averaging an additional one through pollen) (Busch and Delph, 2012). This transmission advantage is opposed by inbreeding depression - a generalised concept for the lower average fitness of selfed progeny. Selfed progeny may have lower fitness for a number of reasons, including reduced genetic diversity, and exposition of harmful recessive alleles (Charlesworth and Charlesworth, 1987). In simple models, inbreeding depression is represented by a value $\delta$ which is the per progeny reduced fitness for a selfed individual. If $\delta>0.5$, the selfer's transmission advantage is outweighed by inbreeding depression, and outcrossing is evolutionarily preferred (Jarne and Charlesworth, 1993): 0.5 is the equilibrium level of inbreeding depression in this model of transmission advantage. This simple relation assumes that selfer pollen is just as successful as outcrosser pollen, ignoring any pollen discounting. Pollen discounting, $0 \leq p \leq 1$, is the reduced relative fitness of pollen spores for selfer pollen (Nagylaki, 1976).

Using Lloyd (1992)'s phenotypic model of selfing versus outcrossing, a non-zero level of pollen discounting results in a frequency dependent equilibrium value for inbreeding depression $\delta$ (Nagylaki, 1976). That is, the maximum level of inbreeding depression required to prevent evolution to selfing varies with the proportion of selfers (explained below with eq. (3)). It can therefore provide a self-perpetuating dynamic to the evolution of selfing, as the level of $\delta$ required to maintain outcrossing increases with the proportion of selfers, so as more selfers evolve, it becomes increasingly more difficult to maintain outcrossing. This means that if environmental circumstances are temporarily in a state that encourages evolution to SC, the proportion of outcrossers may drop below the level at which outcrossing could be maintained even once the environment returns to its previous state. This is a mechanism by which, without any assumed change in inbreeding depression, a temporary environmental disruption may cause one-way transitions to SC.

Our proposed environmental disruption is a temporary limitation of pollen dispersal in the population. This reduces inbound pollen availability to outcrossing plants by an amount $0 \leq l \leq 1$. This limitation also has fitness consequences for selfers, as outbound pollen from selfer plants will be less likely to reach and sire an outcrosser ovule for reproduction. The limitation still has a greater negative effect on outcrossers than selfers, as self-fertilised seeds will be unaffected by the lack of pollen dispersal, while all outcross progeny will be penalised.

\section{The Mathematical Model of Selfing versus Outcrossing}

For general pollen limitation $l$, the initial fitness of outcrossers $W_{x}$ and selfers $W_{s}$ are:

$$
\begin{aligned}
& W_{x}=1-l \\
& W_{s}=1-\delta
\end{aligned}
$$


This corresponds to inbreeding depression for selfers, and inbound pollen limitation for outcrossers. The transmission advantage also needs to be factored in, for which we adapt the model from (Lloyd, 1992). The transmission advantage is the result of an additional crossover process for outcrossers, in which their offspring have an average 50\% chance of transmission of the trait carried by the inbound pollen (rather than their own) (Fisher, 1941). The outcrosser fitness is therefore scaled by $\frac{1}{2}+\frac{1}{2} m_{x}$, where $m_{x}$ is the probability the mate is also an outcrosser. The complement of this amount $\left(\frac{1}{2}-\frac{1}{2} m_{x}\right)$ is added to the selfer fitness (representing those outcrosser progeny transmitting the selfer phenotype). This term, however, is scaled by the relative proportion of outcrossers to selfers and reduced by pollen limitation, as selfers only benefit as much as there are outcrosser ovules available to sire and their pollen can reach them. The comprehensive fitness equations are therefore:

$$
\begin{aligned}
W_{x} & =(1-l) \frac{1}{2}\left(1+m_{x}\right) \\
W_{s} & =\frac{x}{1-x}(1-l) \frac{1}{2}\left(1-m_{x}\right)+1-\delta \\
\text { where } \quad m_{x} & =\frac{x}{x+(1-x)(1-p)}
\end{aligned}
$$

Again, $m_{x}$ is the probability of inbound pollen being outcrosser rather than selfer, incorporating the effect of pollen discounting $p$. The current proportion of outcrossers in the population is $0 \leq x \leq 1$.

From these fitness equations we derive the level of equilibrium inbreeding depression $\delta$, above which outcrossing is evolutionarily preferred, and below which selfing is preferred:

$$
\frac{2 l p x+(1+l)(1-p)}{2(p(x-1)+1)}
$$

Refer to figs. 1(a) and 1(b) for an illustration of this relationship. As we can see, for $0<p<1$, the level of inbreeding depression required to maintain outcrossing increases with selfer proportion (ie. with decreasing $x$ ); selfers have a greater advantage as the selfer proportion increases. Further, the addition of pollen limitation $l=0.2$ in fig. 1(b) shifts the curves upward, giving selfers a selective advantage over the $l=0$ condition. The curves are also contracted in the vertical $(\delta)$ dimension, making this difference more pronounced at higher levels of $p$. We use changes in the value of $l$ to exhibit temporary environmental conditions that favour selfing.

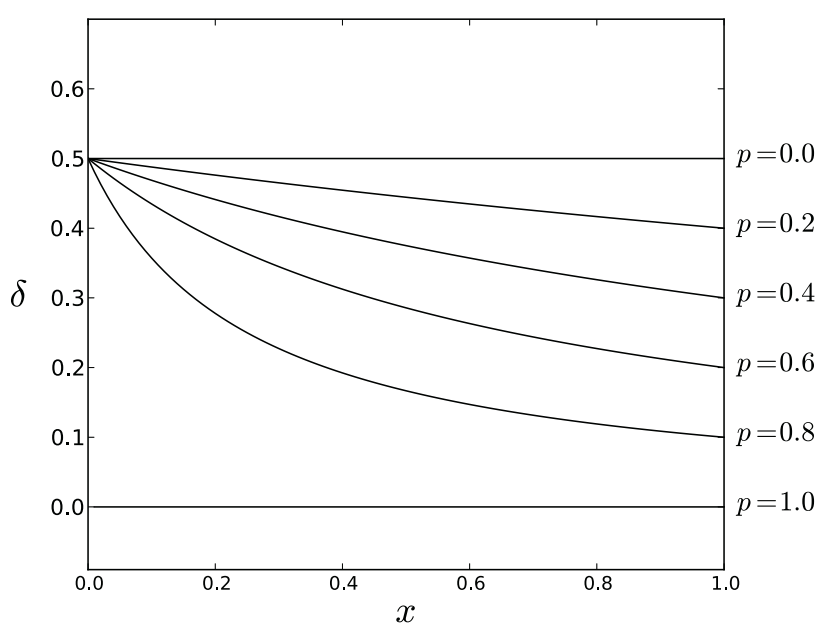

(a) Equilibrium $\delta$ without pollen limitation $(l=0)$

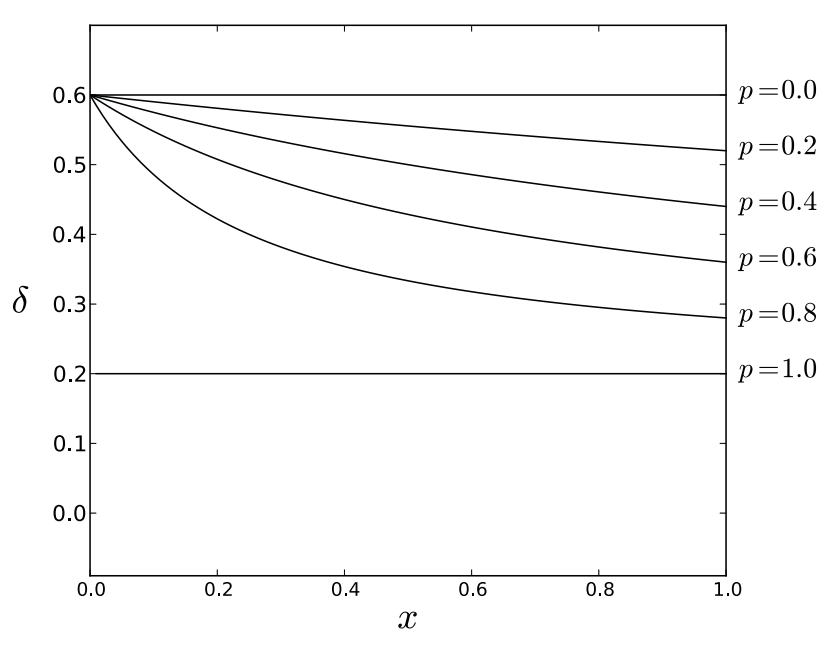

(b) Equilibrium $\delta$ under pollen limitation $(l=0.2)$

Figure 1: Equilibrium inbreeding depression $\delta$ at outcrosser proportion $x$ for different values of pollen discounting $p$, with 1(b) and without 1(a) pollen limitation $l$ (see eq. (3)).

We present two alternative models. In the first, Model A, the transition rate is caused exclusively by the arrival and fixation of the selfer mutation, under conditions that constantly favour selfing. In Model B, conditions generally favour outcrossing, but there are environmental disruptions, occurring with a certain rate $r$, that limit $l$ the pollen dispersal for some duration $d$, during which the conditions favour selfing. This second model, the environmental model, will require a higher mutation rate than the first, as selfer morphs are only favoured by natural selection during disruptions, rather than constantly. The final point of differentiation for the two models, then, will be the mutation rate required to achieve the empirically observed transition rate, given the 
background assumptions of the models.

\section{Methods}

As stated, the target is an average transition rate of $0.555 /$ million years. We run many repeats $(500)$ of a single population under both conditions (original Model A and environmental disruption Model B), recording the number of years it takes to transition to selfing each time. We then take the reciprocal of the mean length of time, arriving at the average transition frequency. After fixing certain parameters of the models, we search manually for parameters that produce the target transition rate for these conditions. Using the same criterion as Goldberg et al. (2010), we conservatively classify a species as SI as long as it is not completely SC, ie. no polymorphism, approximated as less than $1 \%$ of the SI phenotype present in the population; a transition is said to occur when the outcrosser proportion goes below 0.01 .

The simulation is a genetic algorithm with a single population, initially fully outcrossing $(x=1)$. We use roulette selection, which is equivalent to a diffusion approximation of selection and drift (Cherry and Wakeley, 2003). The fitness values for outcrossers and selfers are as per eqs. (1a) and (1b).

Upon selection, if the phenotype is selfer, it is added to the next generation, but if outcrosser, it is combined with pollen from another plant in the population. The probability that this mate is an outcrosser, as opposed to selfer, is $m_{x}$ (eq. (2c)).

The phenotype that goes into the next generation is from either the selected plant or the mate, with equal probability. This is equivalent to the average effect of crossover for outcrossing plants. The net effect of this selection and probabilistic recombination process is captured in fitness eqs. (2a) and (2b). The trait is also probabilistically mutated according to the (phenotypic, per gene per generation) mutation rate $\mu$ before being added to the next generation.

After each generation, we check if the population has transitioned to SC $(x<0.01)$ and break out of the current run if this is the case, recording the length of time that has passed. One generation is equal to one year, a working value used by other models of plants in the Solanaceae family (Vekemans and Slatkin, 1994). For a high level overview of the computer simulation's operation, refer to algorithm 1 .

\section{Parameters}

Table 1 shows the initial set of parameters for the models. The effective population size $N_{e}$ for Solanaceae does vary, but 6000 is within the expected range (Richman et al., 1996). A conservative level of pollen discounting, 0.2, has been chosen initially. As explained, Model A requires that the conditions favour selfing constantly, so a value of $\delta=0.3$ has been chosen to fulfil this requirement (see fig. 1(a), 0.3 is below the $\delta$ equilibrium for $p=0.2, l=0$ ). For Model $\mathrm{B}$, the environmental disruption model, we need selective

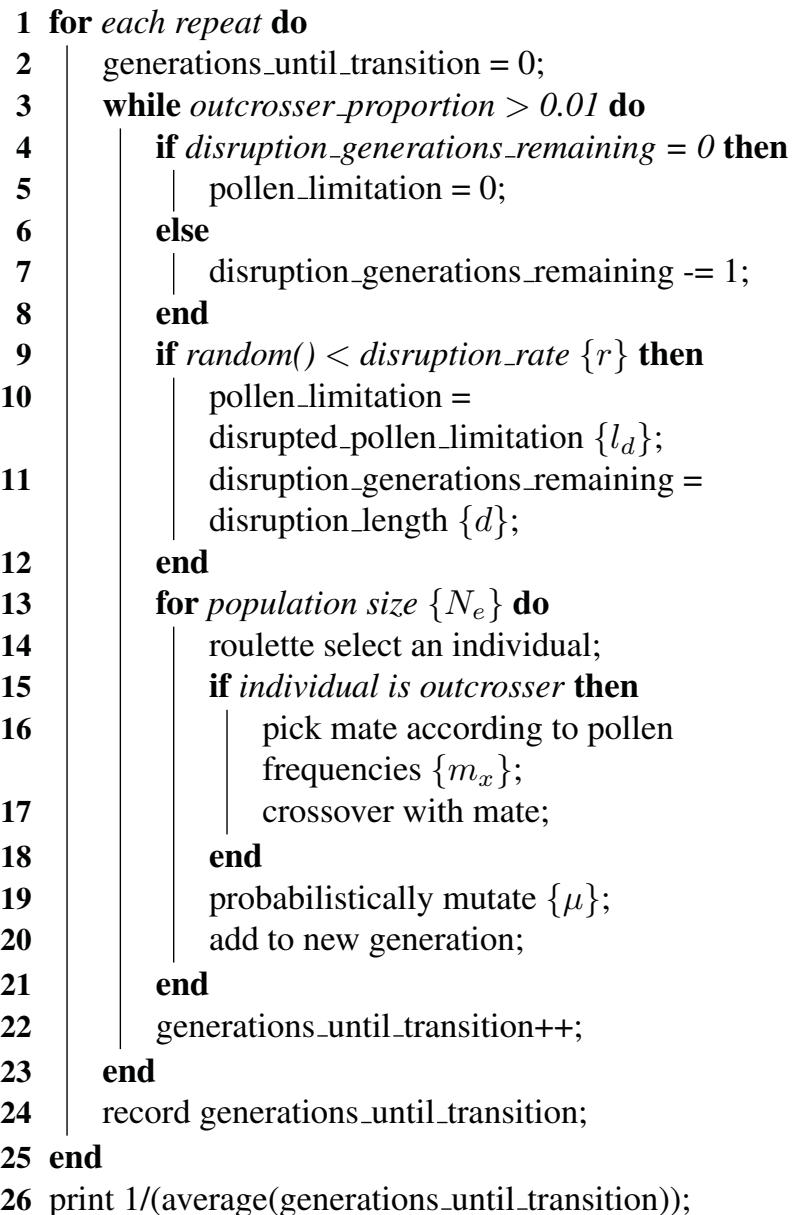

Algorithm 1: Model algorithm

neutrality or favoured outcrossing under background pollen limitation $l=0$ (fig. 1(a), 0.5 is above the $\delta$ equilibrium for $p=0.2, l=0$ ), and favoured selfing under the disruption condition (fig. 1(b), 0.5 is below the $\delta$ equilibrium for $p=0.2, l=l_{d}=0.2$ ).

\begin{tabular}{c|c|c|c} 
& Parameter description & Model A & Model B \\
\hline$p$ & Pollen discounting rate & \multicolumn{2}{|c}{0.2} \\
\hline$l$ & Background pollen limitation & \multicolumn{2}{|c}{0} \\
\hline$l_{d}$ & Pollen limitation (disrupted) & N/A & 0.2 \\
\hline$N_{e}$ & Effective population size & \multicolumn{2}{|c}{6000} \\
\hline$\delta$ & Inbreeding depression & 0.3 & 0.5 \\
\hline$\mu$ & Mutation rate & $*$ & $*$ \\
\hline$r$ & Disruption rate, /species/generation & 0 & $*$ \\
\hline$d$ & Disruption duration, generations & 0 & $*$
\end{tabular}

Table 1: Parameters of the model under Models A and B. Values to be found or manipulated are marked by *. 


\section{Results}

In the first section we present the results from Model A, the model under which species selection directly opposes constant individual incentive for selfing, and Model B, where environmental disruptions bring about temporary individual incentive for selfing. We indicate parameter values under which these alternative low level models exhibit the empirically observed transition rate of 0.555 per lineage per million years $(0.555 \mathrm{E}-6)$ at the species level. We then go on to present some typical evolutionary trials, exposing the underlying selection mechanics of the models.

\section{Results from Models A and B}

In each case, the resultant transition rate is the mean frequency of transition over 500 trials of the single population genetic algorithm. Parameter values were found by manual experimentation given the fixed values established by the model assumptions, detailed previously in table 1 . The output parameter of interest is the phenotypic mutation rate required under each model to bring about the rate of transition observed by Goldberg et al..

\begin{tabular}{|c|c|c|c|c|}
\cline { 2 - 5 } \multicolumn{1}{c|}{} & $\boldsymbol{\mu}$ & $\boldsymbol{r}$ & $\boldsymbol{d}$ & Transition rate \\
\hline $\mathbf{A}$ & $5.17 \mathrm{E}-10$ & 0 & 0 & $0.547 \mathrm{E}-6$ \\
\hline $\mathbf{B}_{0}$ & $1.33 \mathrm{E}-8$ & $\langle 1 \mathrm{E}-5\rangle$ & 5000 & $0.563 \mathrm{E}-6$ \\
\hline $\mathbf{B}_{1}$ & $2.17 \mathrm{E}-8$ & $\langle 1 \mathrm{E}-5\rangle$ & 3000 & $0.537 \mathrm{E}-6$ \\
\hline $\mathbf{B}_{2}$ & $2.17 \mathrm{E}-7$ & $\langle 1 \mathrm{E}-5\rangle$ & 500 & $0.552 \mathrm{E}-6$ \\
\hline $\mathbf{B}_{1}$ & $2.17 \mathrm{E}-8$ & $1 \mathrm{E}-5$ & $\langle 3000\rangle$ & $0.537 \mathrm{E}-6$ \\
\hline $\mathbf{B}_{3}$ & $2.28 \mathrm{E}-8$ & $5 \mathrm{E}-6$ & $\langle 3000\rangle$ & $0.567 \mathrm{E}-6$ \\
\hline $\mathbf{B}_{4}$ & $2.33 \mathrm{E}-8$ & $1 \mathrm{E}-6$ & $\langle 3000\rangle$ & $0.572 \mathrm{E}-6$ \\
\hline
\end{tabular}

Table 2: Parameters and results under Model A (original, no disruption: $r, d=0$ ) and $\mathbf{B}$ (temporary environmental disruptions: $r, d>0$ ). Transition rate should approximate $0.555 \mathrm{E}-6$. The table is grouped, where values held constant are shown in angle brackets, while others were manipulated to obtain the target transition rate. Result $\mathrm{B}_{1}$ is repeated in the third group for convenient comparison.

Table 2 shows that a transition rate of approximately $0.555 \mathrm{E}-6$ can be obtained under multiple conditions; either model is able to potentially explain the empirical observations, but with a different necessary value for the mutation rate $\mu$. For Model A, the background assumptions are such that there is only one possible value, found to be 5.17E-10. Under Model B there is more scope for interaction between the parameters during the search. Holding the disruption rate $r$ at an average of once per 100,000 years (1E-5), rows $\mathrm{B}_{0}, \mathrm{~B}_{1}$, and $\mathrm{B}_{2}$ show that higher mutation rates are required for shorter durations of disruption. Keeping the disruption duration $d$ at 3000 years, we similarly see from rows $\mathbf{B}_{1}, \mathbf{B}_{3}$, and $\mathrm{B}_{4}$ that lower values of disruption rate $r$ require higher mutation rates, but the effect is considerably less significant.
The required mutation rate is more sensitive to the duration of the disruptions than their frequency.

\section{Example Evolutionary Trials}

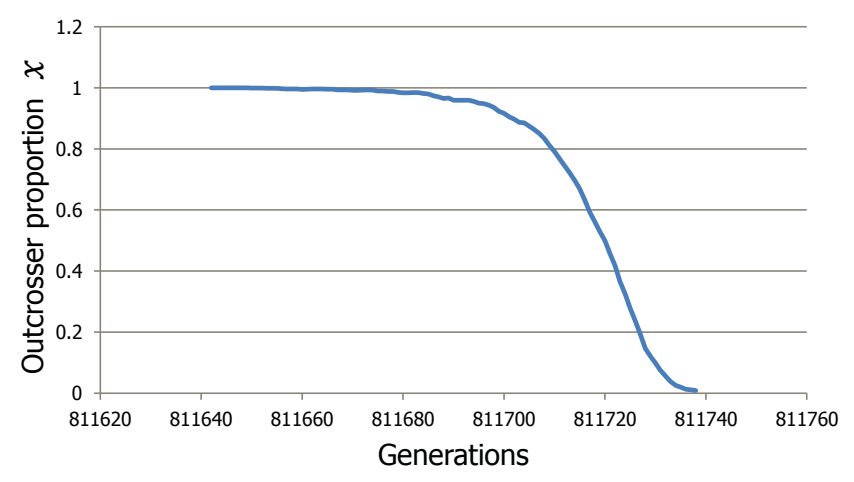

(a) A final phase of the evolution curve once selfing manages to spread, typical under both models.

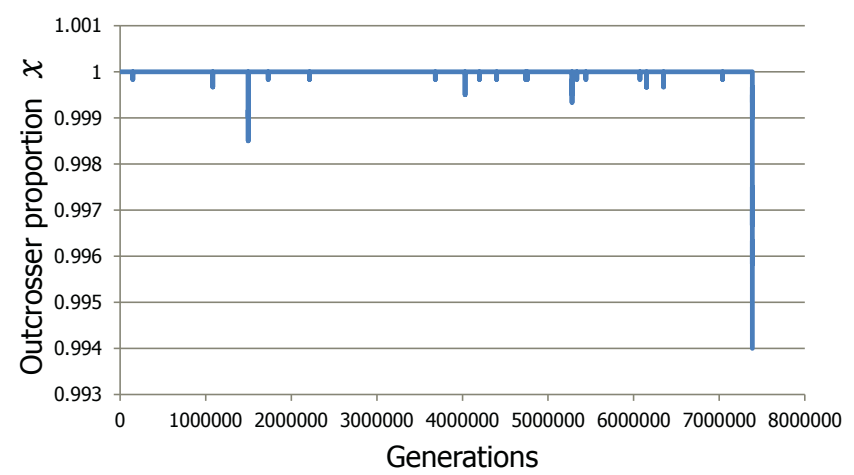

(b) An example evolution curve under Model A where SC mutations arise and are lost multiple times under drift before managing to spread and fixate. The final line down on the right continues to full selfing as in fig. 2(a). Note the scale of the y axis: $x$ does not get below 0.9985 without SC spreading.

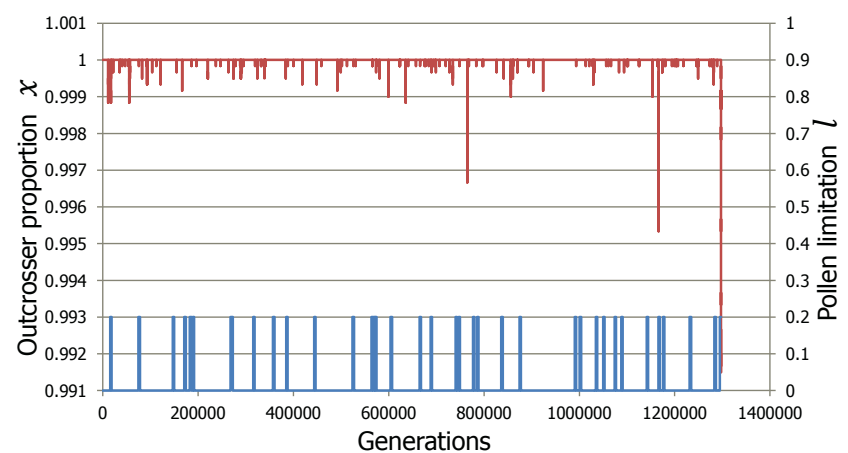

(c) An example evolution curve under Model B. The level of pollen limitation, alternating between 0 and 0.2 on the secondary y axis due to disruption, is also shown. Observing the scale of the primary $\mathrm{y}$ axis, $x$ (the top curve) reaches below 0.9965 without SC managing to spread, lower than under Model A in fig. 2(b). The final line down on the right again continues to full selfing as in fig. 2(a).

Figure 2: Example sequences from typical evolutionary runs. 
Under Model B, the temporary disruptions in the environment allow SC mutations to arise and begin to spread if they are not lost by drift, and may get further than is typical under Model A. This is illustrated in figs. 2(b) and 2(c), where the different scales of the y axis show that the outcrosser proportion can typically get slightly lower in B without a full $\mathrm{SC}$ invasion. This is likely due to the inconsistent selection pressure provided by Model B, as disruptions are brought in and out, shifting the balance of fitness towards and away from outcrossing over time.

Both models produce similar final phases of SC spreading to fixation, as seen in fig. 2(a), as under either model, once selfers reach a certain proportion, selection pressure becomes reinforcing and full invasion becomes highly probable.

\section{Discussion}

Evolutionary models that consider the interaction between multiple levels of the biological hierarchy provide a complex challenge. We have taken Goldberg et al.'s species level empirical data and attempted to realise the individual level processes that give rise to the SI-to-SC species transition rate. Using a genetic algorithm and Lloyd (1992)'s phenotypic model, we discover the mutation rates required under two alternative models, given certain assumptions.

We begin to explore the conditions under which the target transition rate can be produced, and show that there seems to be scope for an environmental model to help explain the evolutionary history of SI and SC in the Solanaceae plant family. Assessing the likelihood of the presented model, or of alternative environmental variation hypotheses, will come down to the plausibility of the required mutation rates. If the mutation rate required of Model A, under the pure species selection hypothesis, is too low, this may suggest individual selection is a significant factor, mediated by environmental conditions. Our Model B presents one such possibility.

The method presented of separating out the individual selection process from the species level process may be applicable to other questions regarding multi-level selection processes. By starting with empirical evidence at the species level and reverse engineering the individual selection pressure using established models, we can explore the real world parameter ranges required to meet alternative theories. These parameters can then hopefully be subject to empirical test, to observe which model obtains.

We did not have time to perform more comprehensive parameter sweeps to provide a robustness analysis. Investigating the relationships between the sets of parameters may prove fruitful as well.

In future work, alternative theories of environmental variation should be explored. In the first instance, an alternative take on Model B would be to have pollen limitation $l$ vary continuously in the background, rather than being manipulated by binary disruption events. It may be that gradual or shallower yet longer dips in dispersal can produce similar rates of transition, for example. More complex models of inbreeding depression and pollen discounting should also be incorporated, as unforeseen interactions between environmental variation and fitness over time may be exhibited.

In summary, we have examined within-species dynamics, under individual selection, that can account for the species level rate of transition that has been empirically observed. Given certain conditions, we obtained the values necessary for the mutation rate to explain the data under two alternative models. Individual based modelling techniques were effectively employed, enabling the analysis of these stochastic models under environmental interaction. By attempting to establish the details of the biological interactions below the species level, we indicate parameter values that may support or reject the original species selection hypothesis.

\section{References}

Barrett, S. C. (1988). The evolution, maintenance, and loss of selfincompatibility systems. Plant reproductive ecology: patterns and strategies, pages 98-124.

Brooks, R. A. (1991). Intelligence without representation. Artificial intelligence, 47(1):139-159.

Busch, J. and Delph, L. (2012). The relative importance of reproductive assurance and automatic selection as hypotheses for the evolution of self-fertilization. Annals of botany, 109(3):553-562.

Caballero, A. et al. (1994). Developments in the prediction of effective population size. Heredity, 73(6):657-679.

Charlesworth, D. and Charlesworth, B. (1987). Inbreeding depression and its evolutionary consequences. Annual review of ecology and systematics, 18:237-268.

Cherry, J. L. and Wakeley, J. (2003). A diffusion approximation for selection and drift in a subdivided population. Genetics, 163(1):421-428.

Damuth, J. and Heisler, I. L. (1988). Alternative formulations of multilevel selection. Biology and Philosophy, 3(4):407-430.

Di Paolo, E. A., Noble, J., and Bullock, S. (2000). Simulation models as opaque thought experiments.

Fisher, R. (1941). Average excess and average effect of a gene substitution. Annals of Human Genetics, 11(1):53-63.

Franklin-Tong, V. E. (2008). Self-incompatibility in flowering plants: evolution, diversity, and mechanisms. Springer.

Goldberg, E. E., Kohn, J. R., Lande, R., Robertson, K. A., Smith, S. A., and Igić, B. (2010). Species selection maintains selfincompatibility. Science, 330(6003):493-495.

Hamilton, W. D. (1964). The genetical evolution of social behaviour. i. Journal of theoretical biology, 7(1):1-16.

Holsinger, K. (1991). Mass-action models of plant mating systems: the evolutionary stability of mixed mating systems. American Naturalist, pages 606-622. 
Holsinger, K. (1996). Pollination biology and the evolution of mating systems in flowering plants. Evolutionary biology, 29.

Igic, B., Lande, R., and Kohn, J. R. (2008). Loss of selfincompatibility and its evolutionary consequences. International Journal of Plant Sciences, 169(1):93-104.

Jarne, P. and Charlesworth, D. (1993). The evolution of the selfing rate in functionally hermaphrodite plants and animals. Annual Review of Ecology and Systematics, pages 441-466.

Johnson, J. B. and Omland, K. S. (2004). Model selection in ecology and evolution. Trends in ecology \& evolution, 19(2):101108.

Lande, R. and Schemske, D. W. (1985). The evolution of selffertilization and inbreeding depression in plants. i. genetic models. Evolution, pages 24-40.

Lewontin, R. C. (1970). The units of selection. Annual Review of Ecology and Systematics, 1:1-18.

Lloyd, D. G. (1992). Self-and cross-fertilization in plants. ii. the selection of self-fertilization. International Journal of Plant Sciences, pages 370-380.

Nagylaki, T. (1976). A model for the evolution of self-fertilization and vegetative reproduction. Journal of Theoretical Biology, 58(1):55-58.

Okasha, S. (2001). Why won't the group selection controversy go away? The British journal for the philosophy of science, 52(1):25-50.

Okasha, S. (2006). Evolution and the Levels of Selection, volume 16. Clarendon Press Oxford.

Queller, D. C. (1992). Quantitative genetics, inclusive fitness, and group selection. American Naturalist, pages 540-558.

Richman, A. D., Uyenoyama, M. K., and Kohn, J. R. (1996). Allelic diversity and gene genealogy at the self-incompatibility locus in the solanaceae. Science, 273(5279):1212-1216.

Turchin, P. (2013). Complex population dynamics: a theoretical/empirical synthesis (MPB-35), volume 35. Princeton University Press.

Vekemans, X. and Slatkin, M. (1994). Gene and allelic genealogies at a gametophytic self-incompatibility locus. Genetics, 137(4):1157-1165.

Wilson, D. S. (1983). The group selection controversy: history and current status. Annual review of ecology and systematics, 14:159-187. 